\title{
Tumeur odontogène adénomatoïde : à propos d'un cas à localisation mandibulaire associé à une prémolaire incluse
}

\author{
Adenomatoid odontogenic tumour: a case report at mandibular location \\ associate with an impacted premolar
}

HAJER HENTATI' ${ }^{1}$, LAMIA OUALHA ${ }^{1}$, LEÏLA NJIM² ${ }^{2}$ INES KALLEL ${ }^{1}$, JAMIL SELMI ${ }^{1}$

\begin{abstract}
RÉSUMÉ
Une patiente âgée de 20 ans consulte pour une tuméfaction parasymphysaire gauche évoluant depuis quelques mois. L'examen clinique montre une tuméfaction dure et indolore avec, à l'examen endobuccal, un comblement du vestibule allant de la 33 à la 36 avec absence de la 34. Les dents de voisinage sont vivantes. L'examen radiologique révèle une image radioclaire uniloculaire en rapport avec la couronne de la 34 qui est incluse. L'aspect est évocateur d'un kyste péricoronaire. L'étude histologique de la pièce opératoire conclut qu'il s'agit d'une tumeur odontogène adénomatoïde. (Med Buccale Chir Buccale 2008 ; 14: 221-225).
\end{abstract}

mots clés: tumeur odontogène adénomatoïde, kyste dentigère, dent incluse

médecine buccale chirurgie buccale

VOL. $14, \mathrm{~N}^{\circ} 4$ 2008

page 221

\section{SUMMARY}

The adenomatoid odontogenic tumour (AOT) is a relatively rare, benign and non-invasive lesion with slow and progressive growth. It arises from odontogenic epithelium with inductive effects on connective tissue. The follicular type located around the crown and often including part of the root of unerupted tooth may mimic dentigerous cyst. One case of AOT in 20 years old female patient is reported. The lesion was located at the mandible and it was associated at an impacted premolar. The most probable primary diagnosis was dentigerous cyst. After surgical enucleation, histopathologic examination revealed the lesion to be an AOT. (Med Buccale Chir Buccale 2008 ; 14: 221-225).

key words: adenomatoid odontogenic tumor, dentigerous cyst, unerupted tooth 
La tumeur odontogène adénomatoïde (TAO) est une tumeur bénigne rare. La symptomatologie clinique se résume souvent à une tuméfaction osseuse d'évolution lente et non douloureuse. La variante folliculaire, associée à une dent incluse, est la plus fréquente ; elle prête à confusion avec un kyste dentigère. L'examen histologique prend alors toute son importance pour préciser la nature de la lésion. Cet article a pour objectif de décrire les aspects cliniques, radiologiques et histologiques de cette tumeur en présentant un cas à localisation mandibulaire.

\section{OBSERVATION}

Une jeune femme âgée de 20 ans, sans antécédents pathologiques particuliers, consulte pour une tuméfaction parasymphysaire gauche évoluant

médecine

buccale

chirurgie

buccale

VOL. $14, \mathrm{~N}^{\circ} 4$ 2008

page 222 depuis quelques mois. La tuméfaction est totalement indolore, de consistance ferme, déformant la région parasymphysaire dont les téguments gardent un aspect normal.

L'examen endobuccal permet de visualiser un comblement du vestibule allant de la 33 à la 36 (Fig. 1). L'absence de la 34 sur l'arcade s'accompagne d'une dépression gingivale vestibulaire, d'une distoversion de la 33 et d'une mésio-version de la 35. Le test de vitalité de ces deux dents est positif. Toutes les autres dents sont présentes sur les arcades.

Le cliché panoramique montre une lésion radioclaire uniloculaire, à contours nets, s'étendant de la 33 à la 36, entourant la couronne et une partie de la racine de la 34 incluse. L'axe corono-radiculaire de

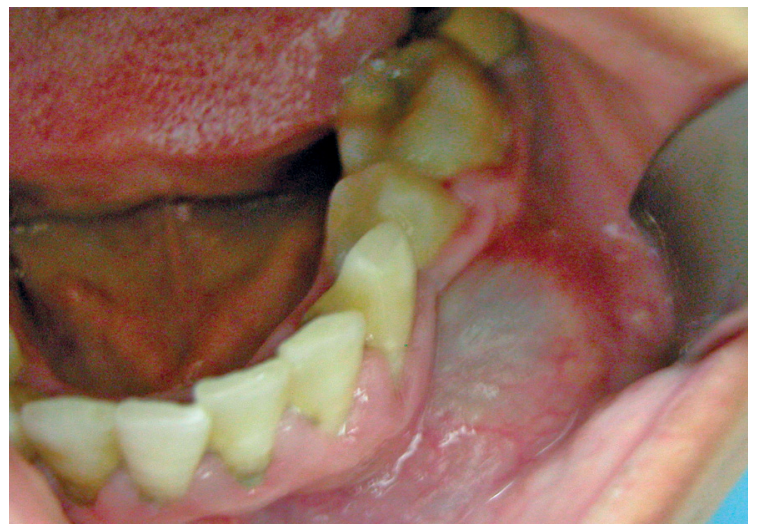

Figure 1 : Vue endobuccale : comblement vestibulaire. Oral view : vestibular deformation.

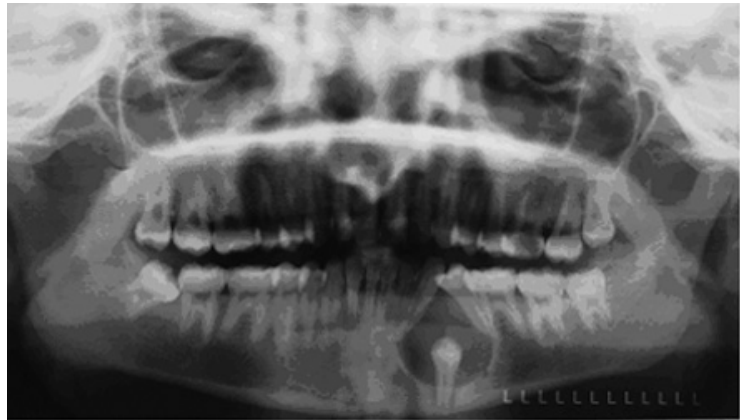

Figure 2 : Radiographie panoramique : image radioclaire et 34 incluse.

Panoramic radiography: radiolucent lesion and 34 impacted.

cette dent est vertical, son apex au contact du bord basilaire. La lésion a entraîné le déplacement des racines de la 33 et de la 35, sans provoquer de résorption (Fig. 2). Initialement, le diagnostic de kyste dentigère en rapport avec la 34 incluse a été évoqué. L'énucléation de cette lésion a été réalisée sans anesthésie locorégionale. L'abord de la lésion a été effectué par une incision intra-sulculaire s'étendant de la 41 à la 36 avec une incision de décharge mésiale à hauteur de la face distale de la 41. Le lambeau muco-périosté a été récliné, découvrant une corticale soufflée. La trépanation de l'os aminci a permis d'exposer la lésion et de réaliser le décollement et l'énucléation de la paroi kystique et l'extraction de la 34. Après révision et curetage de la cavité, le lambeau a été repositionné et suturé. La pièce opératoire était constituée d'une poche de couleur gris-jaunâtre, à laquelle était attachée la 34. Cette dent était presque totalement incluse dans la cavité kystique (Fig. 3). Les suites opératoires ont

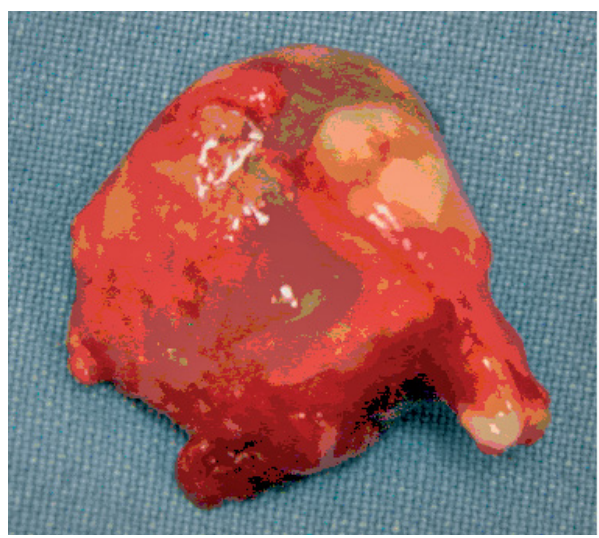

Figure 3 : Pièce opératoire : lésion attachée à la 34 extraite.

Operative view : lesion attached to the extracted 34. 


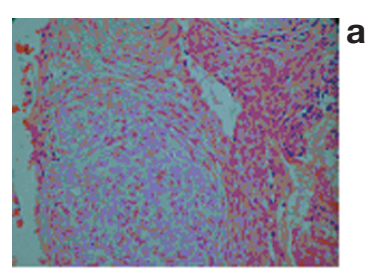

a 西
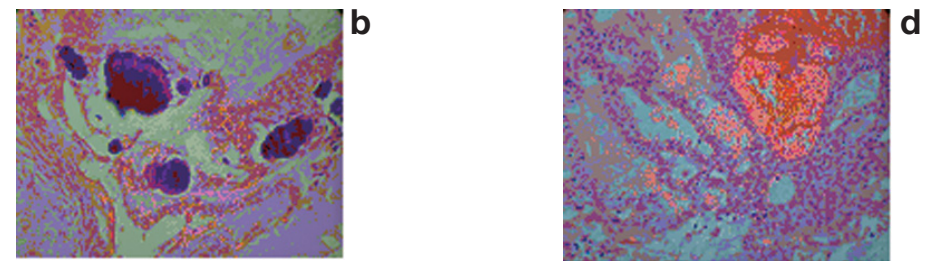

Figure 4 : Aspect histologique. Histologic feature.

4a : cellules basophiles allongées formant des massifs d'aspect tourbillonnant (HE, 2x400).

Spindle-shaped cells that form large whorled aggregates.

$4 b$ : dépôts éosinophiles calcifiés par endroits (HE, 3x400).

Eosinophilic material and calcification.

4c : structures glandulaires tapissées par des cellules cylindriques (HE, x400).

Glandular structures lined by columnar cells.

été simples, sans aucun trouble de la sensibilité labio-mentonnière.

L'étude histologique a révélé que la lésion était en fait une TOA avec une paroi fibreuse épaisse. La lumière de cette formation était tapissée par un épithélium pluristratifié, souvent aplati. Cet épithélium était remplacé, en un endroit, par un bourgeon tumoral composé de cellules basophiles allongées qui s'enroulaient les unes autour des autres, formant des massifs d'aspect tourbillonnant (Fig. 4a). Ceux-ci étaient associés à des travées séparées les unes des autres par des dépôts éosinophiles, homogènes, calcifiés (Fig. 4b). Au sein des massifs, quelques structures glandulaires tapissées de cellules cylindriques étaient présentes (Fig. 4c).

En conclusion, l'étude histologique a infirmé le diagnostic initial et orienté vers le diagnostic de TAO.

\section{COMMENTAIRES}

La TOA est une tumeur bénigne des maxillaires; dans la classification de l'OMS, elle fait partie du groupe des tumeurs odontogènes épithéliales et conjonctives, avec ou sans formation du tissu dentaire dur. Cette lésion naît de l'épithélium odontogène et comporte des structures pseudocanalaires avec présence de phénomènes d'induction d'amplitude variable au sein du tissu conjonctif [1].

La TOA a été probablement décrite pour la première fois par Dreibladt en 1907 [2] mais c'est Stafne en 1948 qui l'a considérée comme une entité distincte parmi les tumeurs odontogènes [3]. C'est une tumeur rare, représentant 3\% des tumeurs odontogènes ${ }^{[4]}$. Ce pourcentage est bien supérieur dans certaines études [5,6]. La TOA survient principalement au cours de la deuxième décennie [7], avec des âges extrêmes allant de 4 ${ }^{[8]}$ et 82 ans ${ }^{[9]}$. Elle affecte plus souvent la femme que l'homme avec un sexe ratio $\mathrm{M} / \mathrm{F}$ de $1 / 2{ }^{[10]}$ à 1/9 [7]. II s'agit d'une tumeur principalement maxillaire qui siège, plus habituellement, dans la région de la canine ou de l'incisive latérale [11] ; la localisation en arrière des prémolaires est exceptionnelle ${ }^{[4]}$. La localisation mandibulaire est deux fois moins fréquente que la localisation maxillaire ${ }^{[1,12]}$. On distingue deux variantes de TOA. Une dite centrale ou centro-osseuse : elle forme la majorité avec un pourcentage de $97 \%$ des cas, dont $73 \%{ }^{[13]}$ sont de type folliculaire, c'est-à-dire associé à une dent incluse. Le type extra-folliculaire, contrairement au type folliculaire, n'est pas associé à une dent incluse. La deuxième variante est dite périphérique ou extra-osseuse; c'est une forme rare ${ }^{[14]}$ qui se traduit cliniquement par une tumeur gingivale ressemblant à une épulis fibreuse [15].

L'association de la TOA à d'autres tumeurs odontogènes a été observée, notamment avec une tumeur épithéliale odontogène calcifiée, un kyste odontogène calcifié et un améloblastome ${ }^{[10,16]}$. Outre ces tumeurs odontogènes, la TOA peut être associé à un kyste folliculaire ${ }^{[14,17]}$.

La découverte d'une TOA est souvent fortuite, soit au cours d'un examen dentaire de routine, soit lors d'un examen radiologique réalisé pour un bilan (traitement orthodontique, retard d'éruption d'une dent...). Parfois, c'est le développe- médecine

buccale chirurgie buccale

VOL. $14, \mathrm{~N}^{\circ} 4$ 2008

page 223 
médecine

buccale

chirurgie

buccale

VOL. $14, \mathrm{~N}^{\circ} 4$ 2008

page 224 ment d'une tuméfaction osseuse, d'évolution lente, souvent isolée et indolore, qui alerte le patient et l'incite à consulter. Cette tuméfaction peut être accompagnée parfois de signes dentaires (déplacements, mobilité des dents voisines, retard d'éruption) [1,2].

La TOA peut se présenter sous plusieurs aspects radiologiques qui peuvent être considérées comme typiques mais non pathognomoniques [8]. La variante folliculaire apparaît comme une image uniloculaire radiotransparente, bien limitée, circonscrite par une paroi radio-opaque associée à une dent incluse, le plus souvent la canine maxillaire permanente ${ }^{[4,12]}$, ressemblant à un kyste dentigère. Pour le cas rapporté, la dent associée à la tumeur était la première prémolaire mandibulaire, ce qui est peu fréquent.

La variante extra-folliculaire se présente comme une lésion radiotransparente se trouvant entre, au-dessus ou superposée aux racines des dents [13]. En fonction de la localisation, elle peut simuler un kyste résiduel, un kyste globulo-maxillaire, un kyste parodontal latéral, un kératokyste, une lésion périapicale, ou même un améloblastome ${ }^{[9,18]}$. Lorsqu'il existe des calcifications (78\% des cas) ${ }^{[18]}$, le diagnostic différentiel fait évoquer un kyste odontogène calcifié ou une tumeur épithéliale odontogène calcifiée. La taille moyenne de la TOA varie entre 2 et 4 centimètres dans plus de $50 \%$ des cas mais peut aller jusqu'à $9 \mathrm{~cm}$ [16]. La variante périphérique (extra-osseuse) est considérée initialement comme une tumeur gin-

\section{RÉFÉRENCES}

1 - Favre-Dauvergne E, Auriol M, Le Charpentier Y. Tumeurs odontogéniques. Encyl Med Chir Stomatologie Odontologie, 22-062-F-10, Editions Techniques, Paris, 1995

2 - Piette E. Pathologie dentaire non carieuse (pp 1244). In: Traité de pathologies buccale et maxillo-faciale. E. Piette, H. Reychler ed : De Boeck-Université, Bruxelles 1991.

3 - Martin-Duverneuil N, Choufa A, Guilbert F, FavreDauvergne E, Roisin-Chausson M-H. Pathologie tumorale (pp 93-94). In: Imagerie maxillo-faciale. N Martin-Duverneuil, J. Chiras ed : Flammarion MédecineSciences, Paris, 1997. givale pouvant s'accompagner d'une érosion de la corticale sous-jacente [18].

Seul l'examen anatomopathologique de la pièce opératoire permet de déterminer la nature exacte de la lésion. Macroscopiquement, la TOA centroosseuse a une consistance qui peut être relativement ferme ou le plus souvent ressembler à une paroi kystique. Microscopiquement, elle est bien limitée ${ }^{[4]}$. Elle présente deux populations cellulaires distinctes : des cellules épithéliales polyhédriques ou fusiformes disposées en cordons ou en volutes, et des cellules épithéliales cuboïdales ou cylindriques formant des structures canaliculaires ${ }^{[15]}$. Les cellules épithéliales sont associées à un tissu conjonctif peu abondant mais bien vascularisé [2]. II existe fréquemment un matériel amorphe éosinophile, PAS positif, parfois calcifié [9]. Des foyers de calcification de formes différentes sont souvent disséminés dans la TOA [2]. Les dépôts éosinophiles correspondent pour certains auteurs à une substance amyloïde-like et pour d'autres à une dentine dysplasique [10]. Le traitement de choix est l'énucléation complète. Pour une TOA de volume important, une marsupialisation suivie de l'énucléation peut être indiquée. La dent incluse associée à la TOA, si elle est en position favorable, peut être conservée et mise sur l'arcade par des moyens orthodontiques [19]. L'évolution est constamment favorable, les récidives sont exceptionnelles ${ }^{[3,20]}$ et aucun cas de transformation maligne n'a été rapporté [11].
4 - Piette E, Hustin J. Tumeurs d'origine dentaire (pp 343345). In: La dent normale et pathologique. E. Piette, M. Goldberg ed : De Boeck Université, Bruxelles, 2001.

5 - Adebayo ET, Ajike SO, Adekeye EO. Odontogenic tumours in children and adolescents: a study of 78 Nigerian cases. J Craniomaxillofac Surg 2002 ; 30 : 267-72.

6 - Sriram G, Shetty RP. Odontogenic tumors: a study of 250 cases in an Indian teaching hospital. Oral Surg Oral Med Oral Pathol Oral Radiol Endod 2008 ; 105 : 14-21.

7 - Philipsen HP, Reichart PA, Siar CH, Ng KH, Lau SH, Zhang $X$, Dhanuthai $K$, Swasdison S, Jainkittivong A, Meer S, Jivan V, Altini M, Hazarey V, Ogawa I, Takata T, 
Taylor AA, Godoy H, Delgado WA, Carlos-Bregni R, Macias JF, Matsuzaka K, Sato D, Vargas PA, Adebayo ET. An updated clinical and epidemiological profile of the adenomatoid odontogenic tumour: a collaborative retrospective study. J Oral Pathol Med 2007 ; 36 : 38393.

8 - Motamedi MHK, Shafeie HA, Azizi T. Salvage of an impacted canine associated with an adenomatoid odontogenic tumor: A case report. Br Dent J 2005 ; $199: 89-90$.

9 - Rousseau A, Morency R, Landry PE. Tumeur odontogénique adénomatoïde. J Dent Québec 1993 ; 30 : 309-11.

13 - Mokni M, Biaggi A, Ragot JP, Auriol M, Le Charpentier Y. La tumeur odontogène adénomatoïde. Une observation à localisation mandibulaire inhabituelle. Arch Anat Cytol Pathol 1994 ; 42 : $181-3$.

11 - Le Breton G. Traité de sémiologie et clinique odontostomatologique (p. 250). Editions Cdp, Paris, 1997.

12 - Swasdison S, Dhanuthai K, Jainkittivong A, Philipsen HP. Adenomatoid odontogenic tumors: an analysis of 67 cases in a Thai population. Oral Surg Oral Med Oral Pathol Oral Radiol Endod 2008 ; 105 : 210-5.

13 - Philipsen HP, Srisuwan T, Reichart PA. Adenomatoid odontogenic tumor mimicking a periapical (radicular) cyst: a case report. Oral Surg Oral Med Oral Pathol Oral Radiol Endod 2002 ; 94 : 246-8.
14 - Takahashi K, Yoshino T, Hashimoto S. Unusually large cystic adenomatoid odontogenic tumor of the maxilla: case report. Int J Oral Maxillofac Surg 2001 ; 30 : 173-5.

15 - Bravo M, White D, Miles L, Cotton R. Adenomatoid odontogenic tumor mimicking a dentigerous cyst. Int J Pediatr Otorhinolaryngol 2005 ; 69 : 1685-8.

16 - Pecheur A, De Clercq D, Reychler H. La tumeur odontogénique adénomatoïde. Rev Stomat Chir Maxillofac $1992 ; 93: 341-4$.

17 - Nonaka CF, de Souza LB, Quinderé LB. Adenomatoid odontogenic tumour associated with dentigerous cyst-unusual case report. Rev Bras Otorhinolaringol 2007 ; $73: 129-31$.

18 - Handschel JGK, Depprich RA, Zimmeramann AC, Braunstein S, Kübler NR. Adenomatoid odontogenic tumor of the mandible: review of the literature and report of a rare case. Head Face Med $2005 ; 1,3$. doi:10.1186/1746-160X-1-3

19 - Shetty K, Vastardis S, Giannini P. Management of an unusually large adenomatoid odontogenic tumor. Oral Oncol Extra $2005 ; 41: 316-8$.

20 - Chuan-Xiang Z, Yan G. Adenomatoid odontogenic tumor: a report of a rare case with recurrence. J Oral Pathol Med 2007 ; 36 : 440-3. \begin{tabular}{l}
$\begin{array}{l}\text { médecine } \\
\text { buccale } \\
\text { chirurgie } \\
\text { buccale }\end{array}$ \\
\hline VOL. $14, N^{\circ} 4$ \\
2008
\end{tabular}

page 225 